\title{
Electronic coupling between the unoccupied states of the organic and inorganic sublattices of methylammonium lead iodide: A hybrid organic-inorganic perovskite single crystal
}

\author{
Gabriel J. Man $\odot,{ }^{1,}{ }^{*}$ Cody M. Sterling $\odot{ }^{2}{ }^{2}$ Chinnathambi Kamal $\odot,{ }^{2,3}$ Konstantin A. Simonov, ${ }^{1, \dagger}$ Sebastian Svanström $\odot,{ }^{1}$ \\ Joydev Acharya $\odot,{ }^{4}$ Fredrik O. L. Johansson ${ }^{1},{ }^{1}$ Erika Giangrisostomi $\odot,{ }^{5}$ Ruslan Ovsyannikov, ${ }^{5}$ Thomas Huthwelker, ${ }^{6}$ \\ Sergei M. Butorin, ${ }^{1}$ Pabitra K. Nayak $\odot,{ }^{4, \$}$ Michael Odelius $\odot,{ }^{2}$ and Håkan Rensmo ${ }^{1, \S}$ \\ ${ }^{1}$ Condensed Matter Physics of Energy Materials, Division of X-ray Photon Science, \\ Department of Physics and Astronomy, Uppsala University, Box 516, Uppsala 75121, Sweden \\ ${ }^{2}$ Department of Physics, Stockholm University, AlbaNova University Center, Stockholm 10691, Sweden \\ ${ }^{3}$ Theory and Simulations Laboratory, HRDS, Raja Ramanna Centre for Advanced Technology, Indore 452013, India \\ ${ }^{4}$ TIFR Centre for Interdisciplinary Sciences, Tata Institute of Fundamental Research, 36/P, Gopanpally Village, \\ Serilingampally Mandal, Hyderabad 500046, India \\ ${ }^{5}$ Institute Methods and Instrumentation for Synchrotron Radiation Research, Helmholtz-Zentrum Berlin GmbH, \\ Albert-Einstein-Straße 15, Berlin 12489, Germany \\ ${ }^{6}$ Paul Scherrer Institut, WLGA/212, Forschungsstrasse 111, 5232 Villigen, Switzerland
}

(Received 3 November 2020; accepted 25 June 2021; published 26 July 2021)

\begin{abstract}
Organic-inorganic halide perovskites have been intensively reinvestigated due to their applications, yet the optoelectronic function of the organic cation remains unclear. Through organic-selective resonant Auger electron spectroscopy measurements on well-defined single-crystal surfaces, we find evidence for electronic coupling in the unoccupied states between the organic and inorganic sublattices of the prototypical hybrid perovskite, which is contrary to the notion based on previous studies that the organic cation is electronically inert. The coupling is relevant for electron dynamics in the material and for understanding optoelectronic functionality.
\end{abstract}

DOI: 10.1103/PhysRevB.104.L041302

In spite of the substantial amount of research effort invested globally, the optoelectronic function of the organic cation in the prototypical hybrid organic-inorganic perovskite (HOIP) methylammonium lead tri-iodide $\left(\mathrm{MAPbI}_{3}\right.$ or MAPI) is still unclear [1,2]. Since the early demonstrations of methylammonium-containing perovskite solar cells, device applications of halide perovskites (HaPs) have transitioned towards the use of mixed-cation (with/without methylammonium) formulations due to concerns about the thermal and chemical stabilities of methylammonium [3,4]. This trend was justified in part by evidence from a multitude of approaches that the organic cation is predominantly an electronically inactive filler [2,5-12]. However, the highest-performing $\mathrm{HaP}$ solar cells typically incorporated some fraction of methylammonium in the absorber layer and hot fluorescence was only observed from lead bromide perovskites with organic cations, suggesting that the organic sublattice plays an opto-

\footnotetext{
*gman@alumni.princeton.edu

${ }^{\dagger}$ Present address: Swerim AB, Kista, Stockholm, 16407, Sweden.

‡pabitra.nayak@tifrh.res.in

§hakan.rensmo@physics.uu.se
}

Published by the American Physical Society under the terms of the Creative Commons Attribution 4.0 International license. Further distribution of this work must maintain attribution to the author(s) and the published article's title, journal citation, and DOI. Funded by Bibsam. electronic role [13-15]. In principle, the comparison between direct or inverse photoelectron spectroscopy measurements and electronic structure calculations should reveal the contributions from the occupied or unoccupied states of the organic and inorganic sublattices $[6,16]$. The occupied states near the valence-band maximum (VBM), relevant for optoelectronic functionality in MAPI and related compounds, have been studied using angle-resolved photoelectron spectroscopy; however, it is unclear if methylammonium-related states are present in the energetic region close to the VBM as no explicit evidence for their presence has been found using nonelement-selective photoelectron spectroscopy $[17,18]$. The unoccupied states have been less commonly investigated; a combination of nonelement-selective inverse photoelectron spectroscopy and density of states (DOS) calculations suggests the unoccupied methylammonium states are energetically positioned near the vacuum level and may not play a role in optoelectronic functionality involving band-edge states $[6,19]$.

In this paper, we investigate both the valence-band and conduction-band states of MAPI via an array of electron spectroscopies performed on clean surfaces of in-vacuum cleaved single crystals, and find evidence for organic-inorganic electronic coupling in the unoccupied states. We have resonantly excited the nitrogen $1 s(\mathrm{~N} 1 s)$ core level to impart organic cation selectivity to the electron spectroscopies. We have performed checks for beam-induced sample damage and ensured the measurements are representative of the intrinsic physics of MAPI. Our nitrogen-projected DOS calculation suggests 
a spectroscopically low intensity of methylammonium states, relative to the iodide states, exists in the valence-band region. Our N $1 s$ resonant photoelectron spectroscopy (RPES) measurements confirm that the intensity of methylammonium states, if present in the valence band, is low, and are consistent with a report which shows a low intensity of methylammonium states near the VBM using a complementary technique [N 1s x-ray emission spectroscopy (XES)] [20]. The lowintensity distribution of methylammonium states may still be relevant for optoelectronic functionality. In the course of performing $\mathrm{N}$ ls RPES, we observe nitrogen core-valencevalence $(\mathrm{N} K V V)$ Auger decay, contrary to the findings of a study which reports $\mathrm{N}$ 1s RPES measurements on MAPI films [21]. Through the use of N 1s resonant Auger electron spectroscopy (RAES), we discover the existence of x-ray excited electron delocalization with a timescale comparable to the $\mathrm{N} 1 s$ core-hole lifetime of $\sim 6 \mathrm{fs}$, demonstrating electronic coupling between the unoccupied states of the organic and inorganic sublattices. Based on the aforementioned findings, we conclude that the occupied states of the organic and inorganic sublattices of MAPI are largely independent but the unoccupied states are significantly coupled, and suggest that the coupling is relevant for slow hot electron cooling.

Beam-induced chemical changes to HaP sample surfaces is a known experimental challenge; the starting composition of a $\mathrm{HaP}$ film has been reported to influence how tolerant $\mathrm{HaP}$ samples are to $\mathrm{x}$-ray irradiation [22,23]. We mitigate this experimental challenge through the use of single crystals, which offer a lower concentration of processing-related defects, and a low photon intensity beamline $\left(\sim 10^{11}\right.$ photons $\left.\mathrm{cm}^{-2} \mathrm{~s}^{-1}\right)$, which necessitates the use of a high-transmission electron spectrometer [24]. Millimeter-scale single crystals of MAPI were grown in solution and characterized with $\mathrm{x}$-ray diffraction (XRD), which confirmed the presence of the tetragonal phase at room temperature and its purity [25,26]. Electron spectroscopy measurements were performed at the synchrotron BESSY II, at the soft x-ray beamline PM4, and end-station LowDosePES [24]. The crystals were cleaved in ultrahigh vacuum (UHV) immediately prior to measurement. All measurements were performed at room temperature in a UHV chamber with a base pressure of $1 \times 10^{-9}$ mbar. Using PES measurements, we have extensively checked that the chemical integrity of the single-crystal surfaces remains intact [26]. Through the use of clean surfaces, we ensured all electron emission arising from $\mathrm{N} 1 s$ core-hole decay can be attributed solely to methylammonium; the N $1 s$ PES spectrum can be fitted with one symmetric peak which we assign to the nitrogen atom in methylammonium. Nitrogen $K$ edge near-edge $x$-ray absorption fine structure (NEXAFS) and ground-state electronic structure calculations were performed on snapshots of the $2 \times 2 \times 2$ supercell geometry sampled for five configurations at regular intervals during the $a b$ initio molecular dynamics (AIMD) simulations, where the initial supercell model was generated from reported lattice parameters [25]. The AIMD simulations were performed with the $\mathrm{CP} 2 \mathrm{~K}$ code at $300 \mathrm{~K}$ and 0 atmosphere (atm) to approximate the experimental conditions [27]. Nitrogen $K$-edge NEXAFS spectra, obtained within the half core-hole transition potential approximation (TPHH), were calculated for each nitrogen present in the supercell [28]. Projected DOS calculations

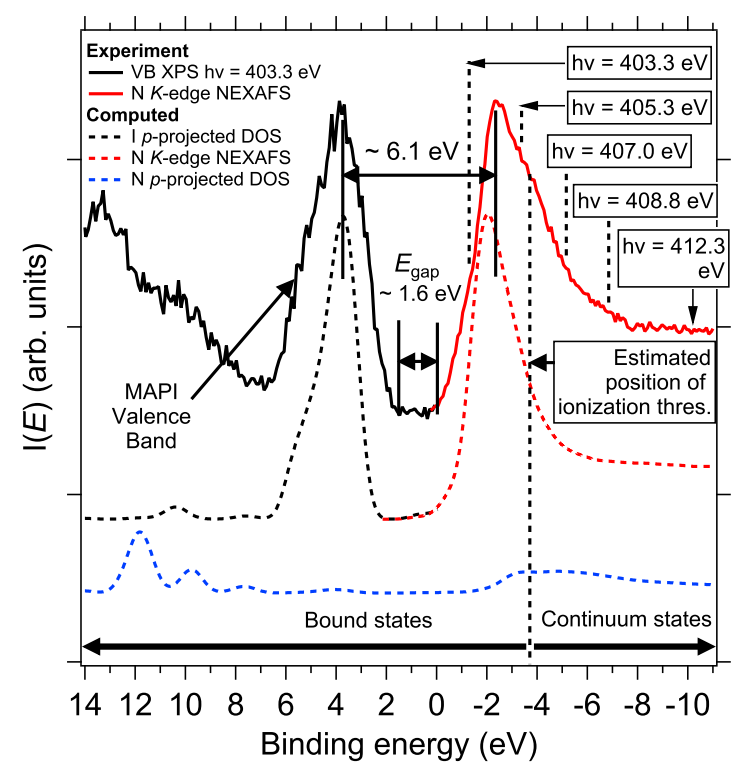

FIG. 1. Experimental vs computed valence photoemission and $\mathrm{N}$ $K$-edge NEXAFS spectra aligned onto a common energy scale. See text for details regarding the energy alignment and the estimation of the energetic position of the ionization threshold. The valence-band peak to NEXAFS peak offset serves as one common reference between experiment and computation. The photon energy labels show the excitation energies used to record detailed autoionization and Auger electron spectra.

were performed using the ground-state wave function. The reader is referred to the Supplemental Material for basic characterization (XRD, surface chemical integrity checks, and characterization with PES) and further experimental and computational details [26].

The complex composition of MAPI implies that elementand orbital-projected DOS calculations are essential for interpreting valence-band photoemission spectra, and the agreement depends on the underlying structural model, the photoionization cross sections, and the modeling of the spectroscopic process [26]. We compare experimental and computed values of the VB peak to NEXAFS peak energy offset, shown in Fig. 1, and simultaneously examine the accuracies of the underlying structural model, the simulated NEXAFS spectrum, and the modeling of the PES spectra in terms of DOS calculations. The experimental spectra consist of the valence-band PES and N $K$-edge NEXAFS spectra. The NEXAFS spectrum was aligned onto the PES energy scale by subtracting the photon energy from the $\mathrm{N} 1 s$ binding energy. The valence-band spectrum of MAPI is similar to a reported spectrum recorded with $h v=500 \mathrm{eV}$ [16]. The computed spectra include the I $p$-projected DOS as a representation of the occupied states, since it comprises the dominant contribution to the valence band, and the computed N $K$-edge NEXAFS (aligned using the computed N $1 s$ orbital energy in the TPHH calculation). The ground-state DOS calculations are consistent with previous reports and serve as an approximation to the photoemission spectra [26]. We observe that the VB peak to NEXAFS peak offsets for the experimental and theoretical spectra are in good agreement, demonstrating that our structural model and calculations are sufficiently 


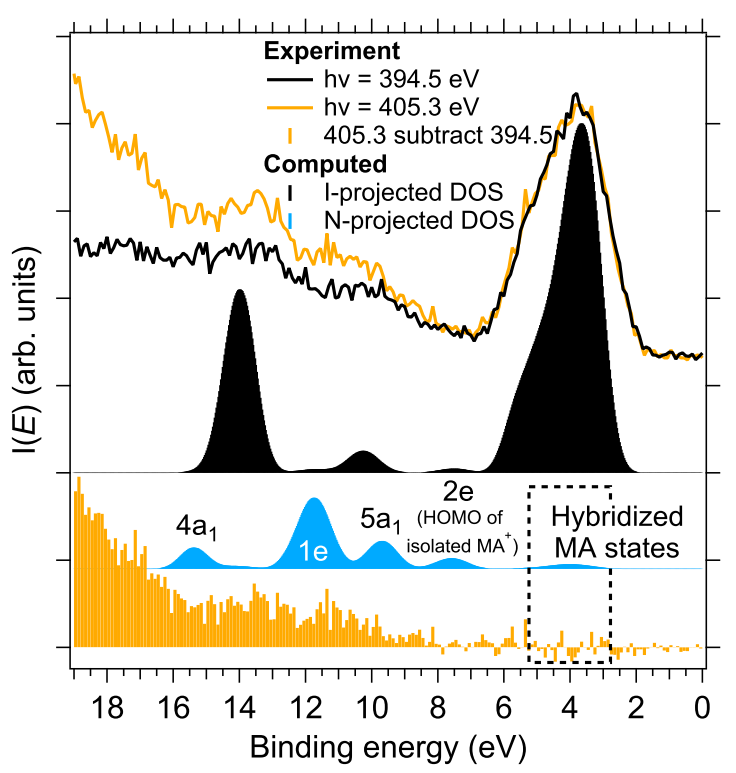

FIG. 2. Nitrogen contributions from the organic sublattice to the electron spectra. The computed DOS is aligned to the valence-band spectra using the I-projected DOS. The subtraction of the offresonant $h v=394.5-\mathrm{eV}$ spectrum from the resonant $h v=405.3-\mathrm{eV}$ spectrum yields a difference spectrum for comparison to the nitrogen-projected DOS.

accurate. Changes to the Fermi-energy position would affect the VB and NEXAFS (via the $\mathrm{N} l s$ binding energy) positions equally. The computed $\mathrm{N} p$-projected DOS of MAPI is displayed also in Fig. 1 as a first approximation to the $\mathrm{N}$ $K$-edge NEXAFS spectrum. Core-hole relaxation effects are manifested as a buildup of resonance intensity towards higher binding energy and a narrowing of the DOS, as evidenced by the comparison between the ground-state nitrogen $p$-projected DOS and the experimental and simulated $\mathrm{N} K$-edge NEXAFS spectra. Other details displayed in Fig. 1 include the reported band gap of MAPI $(\sim 1.6 \mathrm{eV})$ and the energy position of the ionization threshold (or vacuum level), which is estimated from the experimentally determined position of the valenceband maximum and reported values for the band gap (1.6 $\mathrm{eV})$ and electron affinity $(3.6 \mathrm{eV})[6,26]$. Detailed electron spectra were recorded at the five photon energies labeled in the plot. The deduced energy position of the ionization threshold suggests that photoexcitations with energies $\leqslant 405.3 \mathrm{eV}$ are to bound states while photoexcitations with energies $\geqslant 407.0 \mathrm{eV}$ are to continuum states. This deduction is consistent with the observation that the normal Auger spectrum $(h v=412.3 \mathrm{eV})$ is similar to the $h v=407.0 \mathrm{eV}$ spectrum, within signal to noise limitations [26]. Due to its higher signal to background, the $h v=407.0 \mathrm{eV}$ spectrum will be utilized as the normal Auger spectrum. We conclude that the accuracy of our computational work is adequate for interpreting experimental valence photoemission spectra. See Supplemental Material for the reference ground-state DOS calculations and supporting data analyses [26].

In Fig. 2, we present an off-resonant or background spectrum $(h v=394.5 \mathrm{eV})$ and a resonant spectrum $(h v=$ $405.3 \mathrm{eV}$ ), from which a difference spectrum, which highlights all electron emission channels (with different final states) associated with $\mathrm{N} 1 s$ core-hole decay, is extracted. A wider energy region is shown in the Supplemental Material [26]. We compare the $\mathrm{N} l s$ participator autoionization or resonant photoemission portion, of the difference spectrum, to the computed ground-state N-projected DOS. Participator autoionization features are identified by their dispersive nature versus varying photon energy; if plotted on a bindingenergy scale, they remain constant. A comparison of the $h v=405.3 \mathrm{eV}$ (Fig. 2) and $403.3 \mathrm{eV}$ [Fig. S9(c)] difference spectra indicates that similar features in the same $16-0-\mathrm{eV}$ binding-energy region can be seen. To align the N-projected DOS to the valence-band spectra, the I-projected DOS is first aligned in a manner that is consistent with a past report [16]. The nitrogen-projected DOS shows a number of features labeled according to the molecular point group of the methylammonium cation. The highest-occupied molecular orbital of methylammonium is the $2 e$ molecular orbital (MO). The calculations suggest that hydrogen-bonding interaction(s) between methylammonium and its environment results in the formation of states with weak nitrogen character at binding energies close to the VBM. We interpret the origin of these states as hybridization between the organic and inorganic sublattices and note that the intensity of the hybridized states is low relative to the intensities of the other $\mathrm{N}$ features. The hybridized feature has been observed with $\mathrm{N} K$-edge XES, a complementary technique which interrogates the occupied states [20]. The participator autoionization features have a positive slope as a function of increasing binding energy, which qualitatively agrees with the $\mathrm{N}$-projected DOS. Our RPES measurement reveals that the spectroscopic intensity of the hybridized states, if present, is low. Kot et al. performed N 1s RPES measurements on thin films of MAPI and observed nitrogen-related resonant intensity enhancements close to the VBM, though it is unclear what the origin of the nitrogen states is due to the multiple nitrogen species seen in their $\mathrm{N} 1 \mathrm{~s}$ PES spectrum [21]. The overlap of nitrogen and iodide states near the VBM is small, according to our DOS calculations, suggesting that the occupied electronic substructures of the organic and inorganic sublattices are largely independent.

The participator autoionization shown in Fig. 2 comprises a minority fraction of the total $\mathrm{N} 1 s$ core-hole decay. A comparison of the decay spectra arising from excitations to bound ( $h v=403.3 \mathrm{eV})$ versus continuum $(h v=407.0 \mathrm{eV})$ states is shown in Fig. 3; it reveals that the majority of the $\mathrm{N} 1 \mathrm{~s}$ decay is similar (normal Auger decay). This indicates that for the majority of excitations to bound states, the photoexcited electrons have delocalized from their host nitrogen atom and are unable to participate or spectate in the local decay of the $\mathrm{N} l s$ core hole. Possible origins of delocalization include resonant excitation directly into a hybridized and extended (Bloch-like) organic-inorganic state and/or transfer of the excited electron from the organic to inorganic sublattices with a timescale that is clocked by the $\mathrm{N} 1 s$ core-hole lifetime $(\sim 6.3 \mathrm{fs})[29,30]$. Both cases imply that the organic and inorganic sublattices are electronically coupled. We interpret the electron delocalization as charge transfer (CT) from the organic to inorganic sublattices of MAPI for the purpose of estimating a CT time $[31,32]$. We note that the spectral fingerprints of dynamics in the $\mathrm{N}-\mathrm{H}$ bond, a connector between the sublattices, in the intermediate core-ionized 


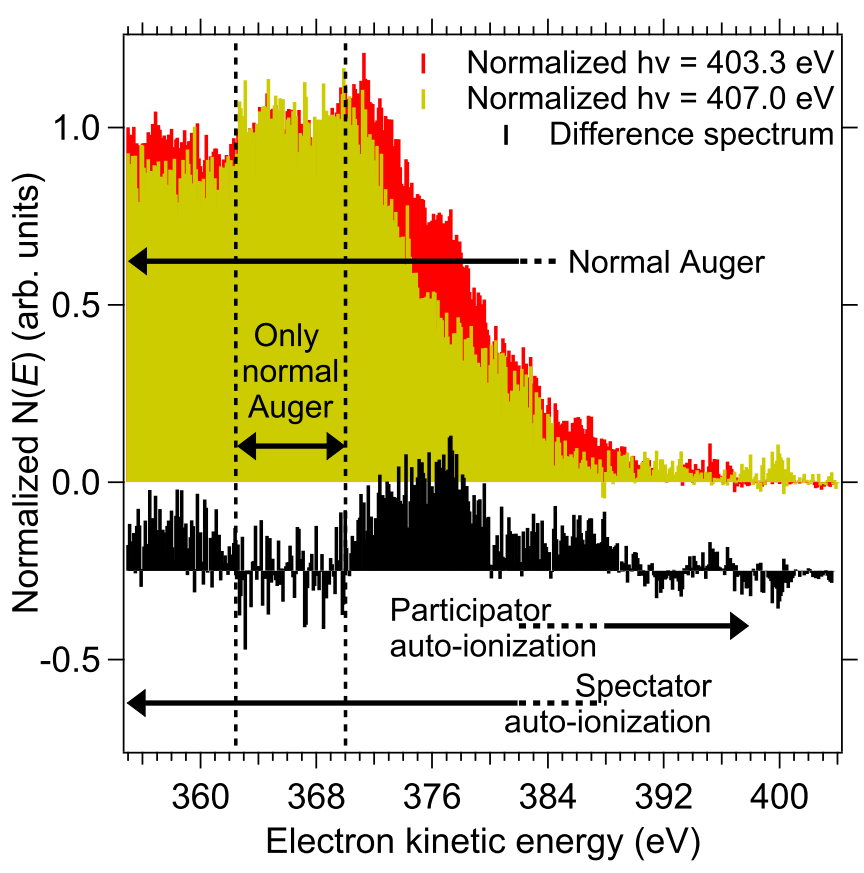

FIG. 3. Nitrogen $1 s$ core-hole decay spectra associated with resonant excitations to bound $(h v=403.3 \mathrm{eV})$ vs continuum ( $h v=$ $407.0 \mathrm{eV}$ ) states. The $h v=407.0-\mathrm{eV}$ spectrum is treated as a normal Auger spectrum (see text). The difference spectrum highlights the autoionization features.

state were reported in a N $K$ XES study of $\mathrm{MAPbI}_{3-x} \mathrm{Cl}_{x}$, which suggests similar dynamics could be present in our RAES measurements involving the core-excited state and affect CT [20]. The relevant decay spectra to examine are the 403.3- and 405.3-eV spectra. The energetic overlap, seen in Fig. 1, between the experimental $\mathrm{N} K$-edge NEXAFS and the bound MAPI empty states (not shown) located between the ionization threshold and the conduction band minimum (CBM) suggests that electron transfer between the organic and inorganic sublattices is possible in the $\mathrm{N} 1 s$ core-excited state. The 403.3-eV spectrum can be regarded as a superposition of the normal Auger spectrum and autoionization features. To highlight the autoionization features in the difference spectrum, we normalize the two spectra utilizing the $\sim 362-$ to $\sim 370-\mathrm{eV}$ region and the background levels at $402 \mathrm{eV}$. We observe that the difference spectrum lacks spectral features in the $\sim 362-$ to $\sim 370-\mathrm{eV}$ region, which indicates that normal Auger decay, the spectral fingerprint of electron delocalization, is present in the $h v=403.3-\mathrm{eV}$ spectrum. We emphasize that this observation holds even if the difference spectrum is generated from the un-normalized 403.3- and 407.0-eV spectra. This interpretation of organic to inorganic sublattice $\mathrm{CT}$ is based in part on the observation that the computed C-, N-, and H-projected DOS overlap in the same energy regions (Fig. S8), which implies that the methylammonium orbitals are fully hybridized and therefore CT from one methylammonium MO to another MO seems unlikely. To estimate the CT time, we assume electron delocalization is a tunneling process with exponential probability as a function of time, the core-hole decay rate is exponential also, and the two processes are independent [30]. The
CT time $\tau_{\mathrm{CT}}=\tau_{N 1 \mathrm{~s}} \times \frac{I_{\text {autoionization }}}{I_{\text {normal auger }}}$, where $\tau_{\mathrm{N} 1 s}$ is the $\sim 6.3$-fs core-hole lifetime of $\mathrm{N} l s$ and $I$ represents the integrated area of autoionization or normal Auger decay, extracted from the $h v=403.3-\mathrm{eV}$ spectrum. The normal Auger energy window for ammonium extends over $\sim 50 \mathrm{eV}$ and the Auger energy window for methylammonium is expected to be similarly wide [33,34]. Given the limited energy windows of the spectra we have recorded, which introduce uncertainties into the modeling of the inelastic background, we provide an order of magnitude estimate of the CT time here. In the range between 362 to $404 \mathrm{eV} \mathrm{KE}$, the integrated area of the normalized $h v=403.3-\mathrm{eV}$ spectrum is 16.2 and the integrated area of the normalized $h v=407.0-\mathrm{eV}$ spectrum is 14.0, with the autoionization features accounting for the difference. The normal Auger component is certainly overestimated since the inelastic background has been included; hence, we do not provide uncertainty estimates. Our $\tau_{\mathrm{CT}}=6.3 \mathrm{fs} \times \frac{2.2}{14}=1 \mathrm{fs}$ estimate establishes a lower bound for this particular excitation $(h v=403.3 \mathrm{eV})$. A similar treatment of the $405.3-\mathrm{eV}$ spectrum indicates that the vast majority of the $\mathrm{N} l s$ decay is Auger and the autoionization features are barely visible, leading to a CT estimate that is $<1 \mathrm{fs}$. Given our two data points, the CT efficiency appears to be positively correlated with photon energy, and one observation of this finding is that the methylammonium- $\mathrm{Pb}, \mathrm{I}$ electronic coupling strength increases with more negative binding energies towards the ionization threshold. The use of RAES for elucidating element-specific features in the conduction band has been demonstrated for other materials. For single-crystal $\mathrm{TiO}_{2}$, crystal-field splitting originating from titanium $d$ states in the conduction band was identified [35]. We conclude that normal Auger decay, the spectral fingerprint of electron delocalization, has been found for resonant excitations to bound states, and if interpreted as CT from the organic to inorganic sublattices of MAPI, leads to an order of magnitude estimate of $1 \mathrm{fs}$ for the CT time. See Supplemental Material for supporting data analyses and a schematic displaying charge transfer, participator, and spectator autoionization processes [26].

We first summarize the findings relevant for organicinorganic electronic coupling in MAPI, then discuss the possible implications for electron dynamics in HOIPs. We demonstrated chemically well-defined single-crystal surfaces of the prototypical HOIP MAPI that are stable under continuous X-ray irradiation and concomitant electron emission for hours. This development provided access to the intrinsic physics of HOIPs. We assessed our computational work to be sufficiently accurate, based on a comparison of the experimental and computed VB peak to NEXAFS peak offsets. The computed nitrogen-projected DOS shows weak features close to the VBM of MAPI which we attribute to hydrogen bonding between the organic and inorganic sublattices. The computed intensity of the hybridized states is low relative to the other features in the N-projected DOS, which may explain why we have not directly observed the states with $\mathrm{N} 1 s$ RPES.

In our investigation of the conduction-band states of MAPI, the element specificity of RAES showed, for resonant excitations to bound states (below the core-ionization threshold), that the photoexcited electrons delocalized from their host nitrogen atoms with a timescale comparable to the $\mathrm{N} 1 s$ core-hole lifetime of $6.3 \mathrm{fs}$. Irrespective of the origin of 
delocalization, the presence of delocalization shows the existence of electronic coupling between the conduction-band states of the organic and inorganic sublattices. By interpreting the delocalization as CT from the x-ray absorption populated states of the organic sublattice to the empty states of the inorganic sublattice, we deduce the lower bound of CT to be $\sim 1$ fs. Charge transfer in the core-excited state, relevant for RAES, may be related to CT in the valence-excited state, relevant for optoelectronic functionality, if the core and valence exciton binding energies are similar. The energies are similar for materials which exhibit efficient core-hole screening such as $\mathrm{C}_{60}[36]$.

Our work motivates further inquiry into the mechanism(s) of electron dynamics in HOIPs. The mechanism of slow hot carrier cooling in HaPs is currently not understood and slow hot carrier cooling has been attributed primarily to slow electron cooling [37,38]. Polaronic effects have been associated with the mechanism of slow hot carrier cooling in HaPs; we note that though polaronic effects exist in $\mathrm{HaPs}$, it is not clear if both the polaronic and potential CT effects can operate concurrently [39-41]. The mechanism underpinning slow hot carrier cooling may be related both to the CT mechanism and the structural dynamics of methylammonium, since the femtosecond charge-transfer time is substantially faster than the two characteristic time constants, $\sim 300$ fs and $\sim 3$ ps, associated with structural reorientation of the methylammonium cation [42].

We thank Helmholtz-Zentrum Berlin für Materialien und Energie for the allocation of beamtime at PM4 (Proposals No. 191-08328 and No. 192-08712). Experiments at BESSY II have been supported by the project CALIPSOplus under the Grant Agreement No. 730872 from the EU Framework Programme for Research and Innovation HORIZON 2020. We acknowledge Svante Svensson (Uppsala), Olle Björne- holm (Uppsala), George Sawatzky (UBC), and Reinhold Fink (Tübingen) for fruitful discussions on modeling the $\mathrm{N} K V V$ AES, and Tomas Edvinsson (Uppsala) for discussions on photoinduced charge transfer. G.J.M. thanks Lucinda Man for the discussion on energy coupling between cubo-octahedron and the octahedron substructures. G.J.M. thanks Sigurd Wagner (Princeton) for the suggestion to investigate the hot carrier cooling mechanisms in lead halide perovskites, and Jeff Schwartz (Princeton) for the discussion on methylammonium deprotonation. G.J.M. thanks Luis K. Ono (OIST) and Yabing Qi (OIST) for their suggestions on how to cleave HaP crystals in vacuum. G.J.M., S.S., K.A.S., and H.R. acknowledge the Swedish Research Council (Grants No. 2018-06465 and No. 2018-04330) and the Swedish Energy Agency (Grant No. P43549-1) for funding. F.O.L.J. acknowledges the Swedish Research Council (Grants No. 2014-6463 and No. 2018-05336) and Marie Skłodowska Curie Actions (Cofund, Project No. INCA 600398) for funding. S.M.B. acknowledges financial support from the Swedish Research Council (Grant No. 2018-05525). C.S., K.C., and M.O. acknowledge support from the European Union's Horizon 2020 Research and Innovation Programme under the Marie Skłodowska-Curie Grant Agreement No. 860553, and the Swedish Energy Agency (Grant No. 2017-006797). The calculations were enabled by resources provided by the Swedish National Infrastructure for Computing (SNIC) at the Swedish National Supercomputer Center (NSC), the High Performance Computer Center North (HPC2N), and Chalmers Centre for Computational Science and Engineering (C3SE) partially funded by the Swedish Research Council through Grant Agreement No. 2018-05973. P.K.N. acknowledges support from the Department of Atomic Energy, Government of India, under Project Identification No. RTI 4007 and Science and Engineering Research Board India Core Research Grant No. CRG/2020/003877.

The authors declare no conflicts of interest.
[1] NREL, Best Research Cell Efficiency Chart (2020), https:// www.nrel.gov/pv/cell-efficiency.html.

[2] D. A. Egger, A. Bera, D. Cahen, G. Hodes, T. Kirchartz, L. Kronik, R. Lovrincic, A. M. Rappe, D. R. Reichman, and O. Yaffe, Adv. Mater. 30, 1800691 (2018).

[3] M. Saliba, T. Matsui, K. Domanski, J.-Y. Seo, A. Ummadisingu, S. M. Zakeeruddin, J.-P. Correa-Baena, W. R. Tress, A. Abate, A. Hagfeldt, and M. Gratzel, Science 354, 206 (2016).

[4] A. Kojima, K. Teshima, Y. Shirai, and T. Miyasaka, J. Am. Chem. Soc. 131, 6050 (2009).

[5] J. M. Frost, K. T. Butler, F. Brivio, C. H. Hendon, M. Van Schilfgaarde, and A. Walsh, Nano Lett. 14, 2584 (2014).

[6] J. Endres, D. A. Egger, M. Kulbak, R. A. Kerner, L. Zhao, S. H. Silver, G. Hodes, B. P. Rand, D. Cahen, L. Kronik, and A. Kahn, J. Phys. Chem. Lett. 7, 2722 (2016).

[7] W.-J. Yin, T. Shi, and Y. Yan, Appl. Phys. Lett. 104, 063903 (2014).

[8] S. Boyer-Richard, C. Katan, B. Traoré, R. Scholz, J.-M. Jancu, and J. Even, J. Phys. Chem. Lett. 7, 3833 (2016).

[9] Y. Rakita, D. Cahen, and G. Hodes, Between Structure and Performance in Halide Perovskites for Photovoltaic Applications:
The Role of Defects (Weizmann Institute of Science, Rehovot, Israel, 2018).

[10] M. Kulbak, D. Cahen, and G. Hodes, J. Phys. Chem. Lett. 6, 2452 (2015).

[11] G. E. Eperon, G. M. Paternò, R. J. Sutton, A. Zampetti, A. A. Haghighirad, F. Cacialli, and H. J. Snaith, J. Mater. Chem. A 3, 19688 (2015).

[12] P. Guo, A. Mannodi-Kanakkithodi, J. Gong, Y. Xia, C. C. Stoumpos, D. H. Cao, B. T. Diroll, J. B. Ketterson, G. P. Wiederrecht, T. Xu, M. K. Y. Chan, M. G. Kanatzidis, and R. D. Schaller, Nat. Commun. 10, 482 (2019).

[13] W. S. Yang, B.-W. Park, E. H. Jung, N. J. Jeon, Y. C. Kim, D. U. Lee, S. S. Shin, J. Seo, E. K. Kim, J. H. Noh, and S. Il Seok, Science 356, 1376 (2017).

[14] Q. Jiang, Y. Zhao, X. Zhang, X. Yang, Y. Chen, Z. Chu, Q. Ye, X. Li, Z. Yin, and J. You, Nat. Photonics 13, 460 (2019).

[15] H. Zhu, K. Miyata, Y. Fu, J. Wang, P. P. Joshi, D. Niesner, K. W. Williams, S. Jin, and X.-Y. Zhu, Science 353, 1409 (2016).

[16] B. Philippe, T. J. Jacobsson, J.-P. Correa-Baena, N. K. Jena, A. Banerjee, S. Chakraborty, U. B. Cappel, R. Ahuja, A. Hagfeldt, 
M. Odelius, and H. Rensmo, J. Phys. Chem. C 121, 26655 (2017).

[17] D. Niesner, M. Wilhelm, I. Levchuk, A. Osvet, S. Shrestha, M. Batentschuk, C. Brabec, and T. Fauster, Phys. Rev. Lett. 117, 126401 (2016).

[18] D. Niesner, APL Mater. 8, 090704 (2020).

[19] S. Tao, I. Schmidt, G. Brocks, J. Jiang, I. Tranca, K. Meerholz, and S. Olthof, Nat. Commun. 10, 2560 (2019).

[20] R. G. Wilks, A. Erbing, G. Sadoughi, D. E. Starr, E. Handick, F. Meyer, A. Benkert, M. Iannuzzi, D. Hauschild, W. Yang, M. Blum, L. Weinhardt, C. Heske, H. J. Snaith, M. Odelius, and M. Bär, J. Phys. Chem. Lett. 12, 3885 (2021).

[21] M. Kot, K. Wojciechowski, H. Snaith, and D. Schmeißer, Chem.: Eur. J. 24, 3539 (2018).

[22] K. X. Steirer, P. Schulz, G. Teeter, V. Stevanovic, M. Yang, K. Zhu, and J. J. Berry, ACS Energy Lett. 1, 360 (2016).

[23] N. Klein-Kedem, D. Cahen, and G. Hodes, Acc. Chem. Res. 49, 347 (2016).

[24] E. Giangrisostomi, R. Ovsyannikov, F. Sorgenfrei, T. Zhang, A. Lindblad, Y. Sassa, U. B. Cappel, T. Leitner, R. Mitzner, S. Svensson, N. Mårtensson, and A. Föhlisch, J. Electron Spectrosc. Relat. Phenom. 224, 68 (2018).

[25] A. Poglitsch and D. Weber, J. Chem. Phys. 87, 6373 (1987).

[26] See Supplemental Material at http://link.aps.org/supplemental/ 10.1103/PhysRevB.104.L041302 for basic characterization (Xray diffraction, surface chemical integrity checks and characterization with PES), computed ground-state DOS, further experimental and computational details and a schematic of the X-ray induced electron emission processes, which includes Refs. [43-60].

[27] J. Hutter, M. Iannuzzi, F. Schiffmann, and J. VandeVondele, Wiley Interdiscip. Rev. Comput. Mol. Sci. 4, 15 (2014).

[28] M. Iannuzzi and J. J. Hutter, Phys. Chem. Chem. Phys. 9, 1599 (2007).

[29] B. Kempgens, A. Kivimäki, M. Neeb, H. M. Köppe, A. M. Bradshaw, and J. Feldhaus, J. Phys. B: At. Mol. Opt. Phys. 29, 5389 (1996).

[30] P. A. Brühwiler, O. Karis, and N. Mårtensson, Rev. Mod. Phys. 74, 703 (2002).

[31] O. Björneholm, A. Nilsson, A. Sandell, B. Hernnäs, and N. Mårtensson, Phys. Rev. Lett. 68, 1892 (1992).

[32] J. Schnadt, P. A. Brühwiler, L. Patthey, J. N. O'Shea, S. Södergren, M. Odelius, R. Ahuja, O. Karis, M. Bässler, P. Persson, H. Siegbahn, S. Lunell, and N. Mårtensson, Nature (London) 418, 620 (2002).

[33] G. L. Gutsev, Theor. Exp. Chem. 21, 9 (1985).

[34] T. Jahnke, U. Hergenhahn, B. Winter, R. Dörner, U. Frühling, P. V. Demekhin, K. Gokhberg, L. S. Cederbaum, A. Ehresmann, A. Knie, and A. Dreuw, Chem. Rev. 120, 11295 (2020).

[35] J. Danger, P. Le Fèvre, H. Magnan, D. Chandesris, S. Bourgeois, J. Jupille, T. Eickhoff, and W. Drube, Phys. Rev. Lett. 88, 243001 (2002).

[36] J. Schnadt, J. Schiessling, and P. A. Brühwiler, Chem. Phys. 312, 39 (2005).
[37] S. Kahmann and M. A. Loi, J. Mater. Chem. C 7, 2471 (2019).

[38] M. Verkamp, J. Leveillee, A. Sharma, A. Schleife, and J. VuraWeis, ChemRxiv (2019).

[39] K. Miyata, T. L. Atallah, and X. Y. Zhu, Sci. Adv. 3, e1701469 (2017).

[40] L. A. D. Irvine, A. B. Walker, and M. J. Wolf, Phys. Rev. B 103, L220305 (2021).

[41] D. Ghosh, E. Welch, A. J. Neukirch, A. Zakhidov, and S. Tretiak, J. Phys. Chem. Lett. 11, 3271 (2020).

[42] A. A. Bakulin, O. Selig, H. J. Bakker, Y. L. A. Rezus, C. Müller, T. Glaser, R. Lovrincic, Z. Sun, Z. Chen, A. Walsh, J. M. Frost, and T. L. C. Jansen, J. Phys. Chem. Lett. 6, 3663 (2015).

[43] Y. Zhang, F. Huang, and Q. Mi, Chem. Lett. 45, 1030 (2016).

[44] O. Schuster, P. Wientjes, S. Shrestha, I. Levchuk, M. Sytnyk, G. J. Matt, A. Osvet, M. Batentschuk, W. Heiss, C. J. Brabec, T. Fauster, and D. Niesner, Nano Lett. 20, 3090 (2020).

[45] J. P. Perdew, Int. J. Quantum Chem. 30, 451 (1986).

[46] J. P. Perdew and M. Levy, Phys. Rev. Lett. 51, 1884 (1983).

[47] C. Motta, F. El-Mellouhi, S. Kais, N. Tabet, F. Alharbi, and S. Sanvito, Nat. Commun. 6, 7026 (2015).

[48] B. Philippe, G. J. Man, and H. Rensmo, in Characterization Techniques for Perovskite Solar Cell Materials, edited by M. Pazoki, A. Hagfeldt, and T. Edvinsson (Elsevier, Amsterdam, 2020), p. 109.

[49] E. A. Kraut, R. W. Grant, J. R. Waldrop, and S. P. Kowalczyk, Phys. Rev. Lett. 44, 1620 (1980).

[50] F. Zu, P. Amsalem, D. A. Egger, R. Wang, C. M. Wolff, H. Fang, M. A. Loi, D. Neher, L. Kronik, S. Duhm, and N. Koch, J. Phys. Chem. Lett. 10, 601 (2019).

[51] F. Zu, C. M. Wolff, M. Ralaiarisoa, P. Amsalem, D. Neher, and N. Koch, ACS Appl. Mater. Interfaces 11, 21578 (2019).

[52] M. Ralaiarisoa, I. Salzmann, F. Zu, and N. Koch, Adv. Electron. Mater. 4, 1800307 (2018).

[53] G. J. S. Man, Metal Oxide/Semiconductor Heterojunctions as Carrier-Selective Contacts for Photovoltaic Applications (Princeton University, Princeton, 2017).

[54] S. Tanuma, C. J. Powell, and D. R. Penn, Surf. Interface Anal. 21, 165 (1994).

[55] C. Quarti, F. De Angelis, and D. Beljonne, Chem. Mater. 29, 958 (2017).

[56] D. Hollas, M. N. Pohl, R. Seidel, E. F. Aziz, P. Slavíček, and B. Winter, Sci. Rep. 7, 756 (2017).

[57] W. Huang, F. Huang, E. Gann, Y.-B. Cheng, and C. R. McNeill, Adv. Funct. Mater. 25, 5529 (2015).

[58] J. A. McLeod, Z. Wu, P. Shen, B. Sun, and L. Liu, J. Phys. Chem. Lett. 5, 2863 (2014).

[59] B. M. Messer, C. D. Cappa, J. D. Smith, K. R. Wilson, M. K. Gilles, R. C. Cohen, and R. J. Saykally, J. Phys. Chem. B 109, 5375 (2005).

[60] M. Ekimova, M. Kubin, M. Ochmann, J. Ludwig, N. Huse, P. Wernet, M. Odelius, and E. T. J. Nibbering, J. Phys. Chem. B 122, 7737 (2018). 